\title{
Isolation and partial characterization of a root- specific promoter for stacking multiple traits into cassava (Manihot esculenta CRANTZ)
}

\author{
M.A. Gbadegesin ${ }^{1}$ and J.R. Beeching ${ }^{2}$ \\ ${ }^{1}$ Department of Biochemistry, Molecular Biology Unit, \\ University of Ibadan, Ibadan, Nigeria \\ ${ }^{2}$ Department of Biology and Biochemistry, \\ University of Bath, Bath, United Kingdom \\ Corresponding author: M.A. Gbadegesin \\ E-mail: magbadegesin@yahoo.com
}

Genet. Mol. Res. 10 (2): 1032-1041 (2011)

Received April 23, 2010

Accepted December 3, 2010

Published June 7, 2011

DOI 10.4238/vol10-2gmr892

\begin{abstract}
Cassava can be cultivated on impoverished soils with minimum inputs, and its storage roots are a staple food for millions in Africa. However, these roots are low in bioavailable nutrients and in protein content, contain cyanogenic glycosides, and suffer from a very short post-harvest shelf-life, and the plant is susceptible to viral and bacterial diseases prevalent in Africa. The demand for improvement of cassava with respect to these traits comes from both farmers and national agricultural institutions. Genetic improvement of cassava cultivars by molecular biology techniques requires the availability of appropriate genes, a system to introduce these genes into cassava, and the use of suitable gene promoters. Cassava rootspecific promoter for auxin-repressed protein was isolated using the gene walking approach, starting with a cDNA sequence. In silico analysis of promoter sequences revealed putative cis-acting regulatory elements, including root-specific elements, which may be required for gene expression in vascular tissues. Research on
\end{abstract}


the activities of this promoter is continuing, with the development of plant expression cassettes for transformation into major African elite lines and farmers' preferred cassava cultivars to enable testing of tissue-specific expression patterns in the field.

Key words: Cassava; Promoters; Gene walking; Gene expression

\section{INTRODUCTION}

The starchy storage roots of cassava provide the staple food for many millions of people in sub-Saharan Africa. In addition, they play a vital role as a famine reserve crop and are becoming increasingly important for processing into higher value products (Montagnac et al., 2009). Cassava can be cultivated on impoverished soils with minimum inputs and is therefore an excellent staple food crop for small hold farmers (Cock, 1985). However, it is far from being an ideal food and crop, as its roots are low in bioavailable nutrients and protein content, contain cyanogenic glycosides, and suffer from a very short post-harvest shelf-life, and the plant is susceptible to viral and bacterial diseases prevalent in Africa (Cock, 1985; Puonti-Kaerlas, 1998; Beeching et al., 1998; Restrepo et al., 2004; Verdier et al., 2004). The demand for the improvement of cassava with respect to these traits comes from both farmers and national agricultural institutions and is articulated through international organisations such as the Cassava Biotechnology Network, IITA and CIAT, together with national institutes, such as Kenya Agricultural Research Institute (KARI), Kenya, National Root Crop Research Institute (NRCRI), Nigeria, and National Agricultural Research Organisation (NARO), Uganda.

For cassava agriculture, it would be ideal to identify and produce solutions to these various problems and to introduce several or most of these improved traits into the major African elite lines and farmers' preferred cultivars. One possible solution is the use of genetic breeding to introduce traits of interest such as disease resistance, extended shelf life (post-harvest), improved protein and micronutrient (e.g., vitamin $\mathrm{A}, \mathrm{Zn}, \mathrm{Fe}$ ) content and low cyanogenic content. However, this is largely not feasible due to the crop's clonal propagation, high heterozygosity and poor flowering ability (El-Sharkawy, 2004). Genetic manipulation through molecular biology techniques is an alternative means to introduce these traits. This approach has been adopted in other crop plants with success and offers opportunities for African crops (Thomson, 2008).

The pyramiding of multiple traits into the major African elite lines and farmers' preferred cassava cultivars may be divided into two aspects: first, how these multiple traits can be readily introduced into a range of different cassava cultivars and, second, how to ensure that all the introduced transgenes are expressed appropriately without interference. It is the second aspect that forms the problem tackled by this project. There is a critical need for root-specific promoters with a range of developmental and tissue specificities within the cassava storage root. Without access to such a toolkit, the transition from research to the release of cassava varieties improved for multiple traits will be delayed. It would be ideal to consider root-specific promoters from cassava, but unfortunately, these are currently largely unknown and uncharacterized. The objective of this project was to isolate and characterize suitable gene promoters from cassava, which could be used to target gene expression in this important food crop. 


\section{MATERIAL AND METHODS}

\section{Plant material and DNA isolation}

Cassava plants (cultivar CM 2177-2) were grown in the tropical glasshouse at the University of Bath at $22-28^{\circ} \mathrm{C}$, relative humidity of $40-80 \%$ and a minimum light period of 12 $\mathrm{h}$ per day under daylight, supplemented with $400 \mathrm{~W}$ Phillips high-pressure sodium lights when necessary. High-quality genomic DNA was extracted from young leaf samples of this cassava cultivar by the method of Dellaporta et al. (1983).

\section{Restriction enzyme digestion and adaptor ligation gene walker library construction}

Separate aliquots (100 ng) of cassava DNA were completely digested with four different restriction enzymes that leave blunt ends, viz DraI, EcoRV, StuI, and PvuII, in a total volume of $100 \mu \mathrm{L}$. Human genomic DNA aliquot was also digested with $P v u \mathrm{II}$ to serve as a positive control. Each batch of digested genomic DNA was purified and then ligated separately to the GenomeWalker ${ }^{\mathrm{TM}}$ adaptor supplied with the GenomeWalker ${ }^{\mathrm{TM}}$ kit (Clontech Laboratories, Inc.). The digestion and ligation of DNA fragments were carried out under the conditions specified by the kit manufacturer. Ligation mixtures were then diluted as specified in the GenomeWalker ${ }^{\mathrm{TM}}$ kit instruction. Each adaptor-ligated, restriction enzyme-digested genomic DNA constitutes a "gene walker library".

\section{Gene-specific primer design and procurement}

Complementary DNA sequences for the cassava auxin repressed-like protein (ARP) gene were mined from the NCBI database (DB 924059). Gene-specific primers were designed from cDNA sequences using the PRIMERW program. The primers were supplied by Invitrogen, UK.

\section{Polymerase chain reaction (PCR)-based gene walking technique}

Two sets of PCR cycles, primary and secondary PCRs, were carried out in sequence. In the primary PCR, $1 \mu \mathrm{L}$ derived from each library was amplified in a PTC 100 Programmable Thermal Controller (MJ Research Inc.), using a set of gene-specific primers (GSP1) and adaptor-specific primer (AP1). The latter was supplied with the GenomeWalker ${ }^{\mathrm{TM}}$ kit (Clontech Laboratories, Inc.) along with the Advantage 2 polymerase enzyme. In the secondary or nested PCR, the products of the primary PCR $(1 \mu \mathrm{L} 50$-fold dilution) were used as templates using nested gene-specific primer (GSP2) and adaptor specific primer (AP2).

\section{Cloning procedures}

Amplified gene promoter was purified using the PCR purification kit (QIAquick) or gel extraction kit (Qiagen). The purified DNA was ligated into pGEM $^{\circledR}$-T Easy vector (Promega) and used to transform competent Escherichia coli DH5 $\alpha$ according to standard procedures (Sambrook et al., 1989). 


\section{DNA sequencing and sequence analyses}

PCR products or recombinant plasmid DNA molecules were sequenced on an ABI 377 automated dye primer sequencer (Applied Biosystems) using GSP2, AP2 or universal primers for the cloning vector. Initial confirmation of sequence identity was performed by BLASTN and TBLASTX searches against the GenBank non-redundant database using default parameters (Altschul et al., 1997). The database of the Plant cis-acting Regulatory DNA Elements (PLACE) (Higo et al., 1999) was used to determine plant cis-acting regulatory elements. Sequence data were assembled with the VECTOR NTI program (Lu and Moriyama, 2004). Where necessary, internal primers were designed and used for further fragment sequencing.

\section{RESULTS AND DISCUSSION}

\section{PCR-amplified cassava promoter regions}

Cassava root-specific promoters required for pyramiding of multiple traits into the major cassava lines and farmers' preferred cassava cultivars are currently largely unknown and uncharacterized. Gene-specific primers were designed using cassava cDNA sequences as template in PCR-based upstream gene walking experiments. Putative cassava gene promoter regions were amplified by primary and secondary PCRs (as described in the Material and Methods section). Figure 1 represents the amplification of the promoter region of a putative ARP gene. The primary PCR produced many DNA bands, which were reduced to few or even a single band in the secondary PCR. In this way, gene specific amplifications were favored. Amplified fragments in the size range of 1.5-2.0 kb were sequenced. Sometimes, smaller fragments were also sequenced for comparison with the sequence from larger fragments in multiple sequence alignments. Figure 2 shows the assembly of ARP gene fragment sequences.
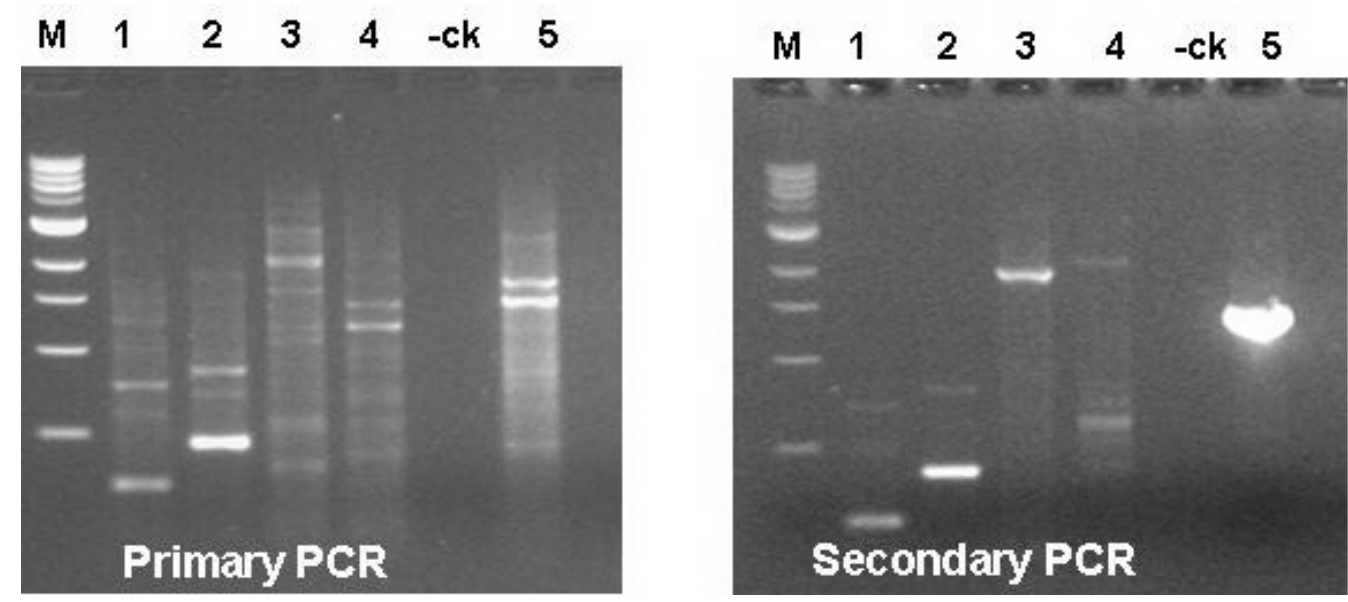

Figure 1. PCR amplification of cassava DNA (promoter regions) for the auxin repressed-like protein gene. M $=1-\mathrm{kb}$ DNA size marker; lane $1=$ DraI; lane $2=E c o \mathrm{RV}$; lane $3=$ StuI; lane $4=P v u \mathrm{II}$; lane $5=$ human $/ P v u \mathrm{II}$ libraries; -ck = negative control with no DNA template. 


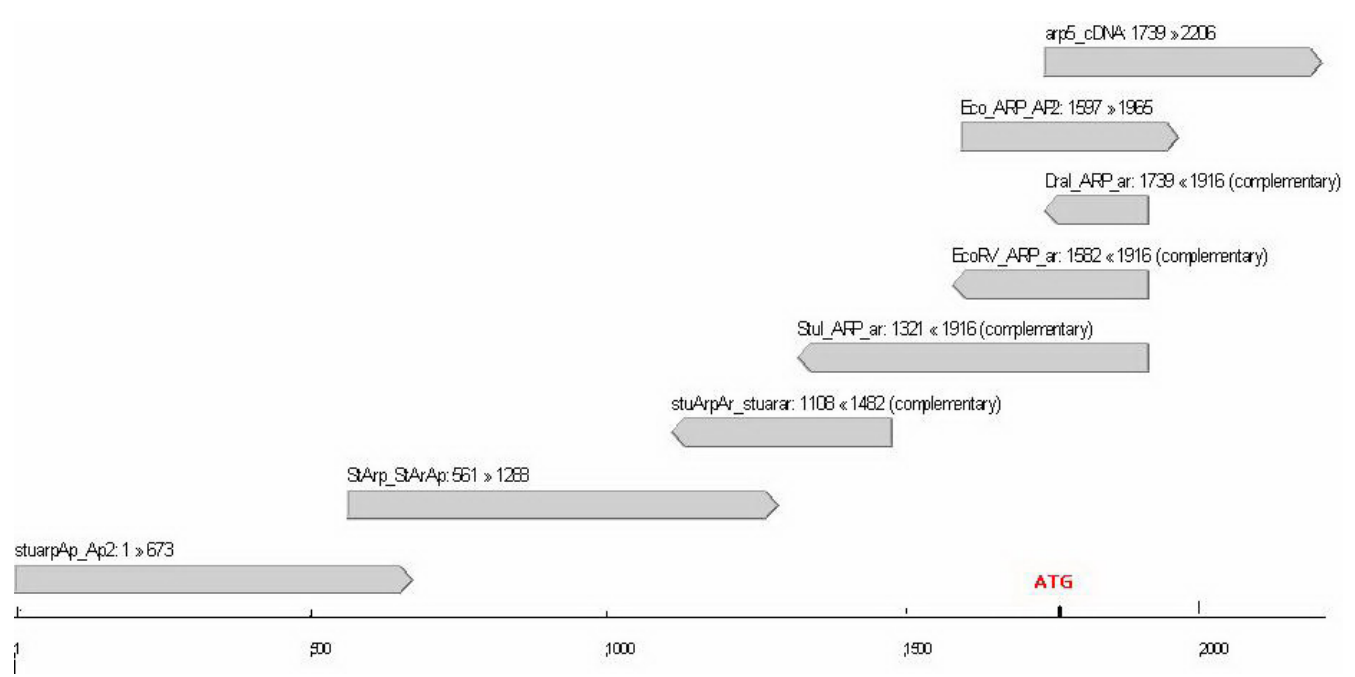

Figure 2. Scheme for the assembly of DNA sequence for auxin repressed-like protein gene. Gene sequence fragments are represented by arrowed head bar showing the $5 \rightarrow 3$ DNA fragment sequence direction. The name of a sequence fragment is a combination of the restriction enzyme for the gene walker library and the sequencing primer. $\mathrm{Eco}=E c o \mathrm{RV} ; \mathrm{Stu} / \mathrm{St}=S t u \mathrm{I} ; \mathrm{ATG}=$ the initiation codon; $\mathrm{ARP}=$ auxin repressed-like protein gene

\section{Sequence analysis of promoter regions}

The sequences of amplified promoter regions were, in the first instance, screened for introns by comparison with the original cDNA sequence. Exon sequences were subjected to BLAST and TBLASTX searches to confirm their identity with the original source cDNA and to identify amino acid sequence motifs. Figure 3 shows the resulting translation of sequences downstream of the start codon, ATG, and the promoter sequence of the putative ARP gene. Amino acid sequence motifs typical of ARP were identified. Sequence analysis of the promoters showed that a TATA box (TATAA) was located 83-bp upstream of the ATG start codon (Figure 3). de Souza et al. (2009) also reported a cassava $\mathrm{Mec} 1$ promoter with the TATA box located 103-bp upstream of the ATG.

\section{Promoter isolation and cloning}

Primers were designed from the consensus sequence derived from the gene walking PCRs to amplify about $1.5 \mathrm{~kb}$ of the promoter sequence upstream of the ATG start codon (see Figure 3). Figure 4 shows the gel picture of the PCR-amplified promoter for the putative ARP gene. Restriction enzyme sites (BamHI and $\mathrm{NcoI}$ ) were incorporated into the primers to facilitate subsequent cloning in the plasmids.

The amplified promoter of the ARP gene was subsequently ligated into pGEM $^{\circledR}$-T Easy vector, and the recombinant plasmid was used to transform competent E. coli DH5 $\alpha$. Plasmids were isolated from bacterial cultures, restriction enzyme digested to size the insert (Figure 5) and sequenced to re-confirm the identity. The insert released after restriction enzyme digestion of the pGEM-T Easy recombinant plasmids was cloned into pCAMBIA 1305 vectors ready for transformation into cassava plants for tissue expression studies. 
AGGACACTPTCGGCGGCAGGTPCGGCGGCCGAAAGTCCCTCCAGAGCCGAAAGTCAG GCAGGTTCGGCGGCACCTPCGGCGGCCGAAACTCCCAGACAGAGACGAAACTCATGC ATGTTCGSCGSCACCPTCGGCGGCCGAAACTGCCAGACAGAGACGAAAGTCTCCTTT CGGGGECAAGTTTCGGCAGCCGAAGGGCTGCCTCCCCAGCCATGTTCGECGGCCGAA AGPTCCITCGGCTGCCGAACCTGGTPTCTGCCAAAGGGCAGAAACTTGGCTCCCAAT GCACATTTCGCCTCCAAACTTATCAAACATGCATCAAACCTATTCTACAACACACAA ACGCAAGCATACATGTTCCTAGGGGTCTCAAACCATCATAAACCCCATCTACAACAC ATCAAGCATCCACATTGTTCAAGAACACACATTATACCCATAAACACAACCATAACC TAAACATGCATTCTAACTCATAGATCTTGCATAAAACTTATTCAAAACATAAAACGA GCITAAGATCGGCTCPTACCTCTPGAAGATCGAGAGAGACGACCCAAAAAACTCGGAG TTGGGAGAGATTTGGTTCTTGAACCTCCAAGCTCCAAAACTTTGCTCAAAAGCTTAA ATCTTCAAAACCAAGTPAAAACAAGTGAAAATCTTGAAAGATPTAGAGGAAGAACAT CAAAAATGGGTGAGGGACGGCGGAGAGCTCACCTGGGCCGAAAATGGGGAAAAGCTC GCCCGTPTTCGGCTAAGGGACCCTTTTATAGTGGCTGGCCAGACCACGTTCGGGGGC CGAATGTGTCTCCGCATGCATGCCATGTTCGGCGGCCSAACCTGGACTTCCCTCACT TATGCTTTCGGGGGCCTAAAGCACACCCECAACGCATGCATGTTCGGCGGCCGAACT TGAGGTTCGACGGCCGAACCTGAGTTTTCCTCCAATGCTATTTTCATGCAAAAACTC ATT'TCTTTCATGCTTAAAACATAAAACACATTAAAATATTTCATAAAAACATGGTT TTACCCTACTAGAGACTTCCGACATCCGAGATTCCACCGGATGGTAGGAATTTCGAT ACCGGAGTCTAGCCGGGTATTACACTCATATAATAGAATTATCATCATTACACGAAA TITCIAGAAGAAAATATGCAATTCCAAAATCITTACTGAAATTGAAAGAGTPTGCTG TCATTTTAGAGACCATGCATAACCCAATTAGTAGGAAGCTTTTACGTTGCTCCCTTC GATTT'TTTT'TCTGCAGATATTTTCTGCAGCCAACTTACCAGCCACTCAAACTAGG ACTACCIAAGTAATATGTATTATTATGCTAAAATAACATCCTAGTACAAAATATTTC ATATTTAAACATTGTTPAAAAATAAATATTTGCAATTCITATCCTGTTTTATAAGAA ATGAAATATAAATTCTCATAGATATTGGATTTGCCACGTGGAACACCCAGACTGGCT ATGGGGCAATAAATGAATCTAAGAACTTGGTGGTCCGAAAGGTGACGCGTAAGATAG CCGACAAAAATCTTGAGCCTCCAATGAAATGAGAGTTCTAGATATCGAGAGATAAGA AGCTTTGTGAAATCCTGGACGTGGGATTTTCAGCAAGATAAGAAAATCTACACTTAA CTCAT AATAAATGCCCACCAAATCCACTTCTCTAATTCACCACTTGAATCAAACAAC ATACAGAGGATTCAAACCCACACTCCTITAAACA

1745 ATGgcagcctcaacaatggcectttcctccecttctcttgceggc

$$
\begin{array}{lllllllllllllll}
\mathbf{M} & \mathbf{A} & \mathbf{A} & \mathbf{S} & \mathbf{T} & \mathbf{M} & \mathbf{A} & \mathbf{L} & \mathbf{S} & \mathbf{S} & \mathbf{P} & \mathbf{S} & \mathbf{L} & \mathbf{A} & \mathbf{G}
\end{array}
$$

1790 aaggcagtgaagctcacccctctgcccctgagctcatgggcaat

$$
\begin{array}{llllllllllllllll}
\mathbf{K} & \mathbf{A} & \boldsymbol{\nabla} & \mathbf{K} & \mathbf{L} & \mathbf{T} & \mathbf{P} & \mathbf{S} & \mathbf{A} & \mathbf{P} & \mathbf{E} & \mathbf{L} & \mathbf{M} & \mathbf{G} & \mathbf{N}
\end{array}
$$

1835 ggccgtttttcaatgaggaaaactgccagcaaggctgtttcctct

$$
\begin{array}{lllllllllllllll}
G & \mathbf{R} & \mathbf{F} & \mathbf{S} & \mathbf{M} & \mathbf{R} & \mathbf{K} & \mathbf{T} & \mathbf{A} & \mathbf{S} & \mathbf{K} & \mathbf{A} & \boldsymbol{\nabla} & \mathbf{S} & \mathbf{S}
\end{array}
$$

1880 ggaagcccatggtacggtccagaccgtgttaagtacttgggtcca

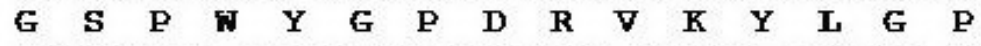

1925 ttctctggtgagcecccatcctacttgactggcgaattccc 1965

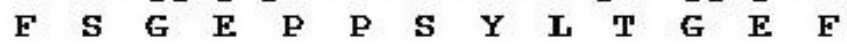

Figure 3. DNA sequence translation/analysis of auxin repressed-like protein gene promoter region. Typical ARP protein amino acid sequence motifs are shown. The initiation codon (ATG) and TATAA sequence typical of a eukaryotic promoter are shown in bold and underlined. 


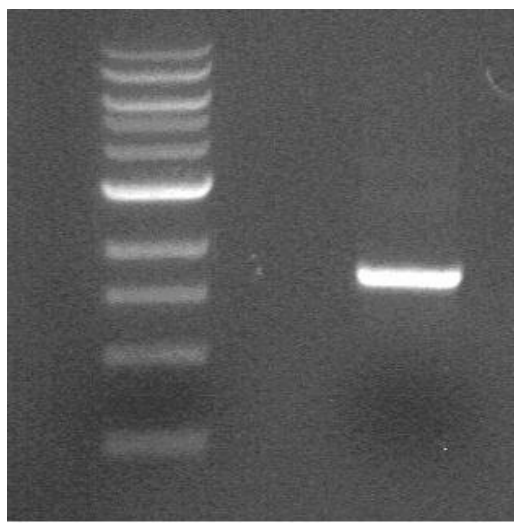

Figure 4. PCR amplification of cassava auxin repressed-like protein gene promoter. The primers were designed to amplify just the promoter (ATG inclusive). The marker shown is New England Biolabs 1-kb ladder (size details in Figure 1). About 1.5-kb size promoter was isolated.

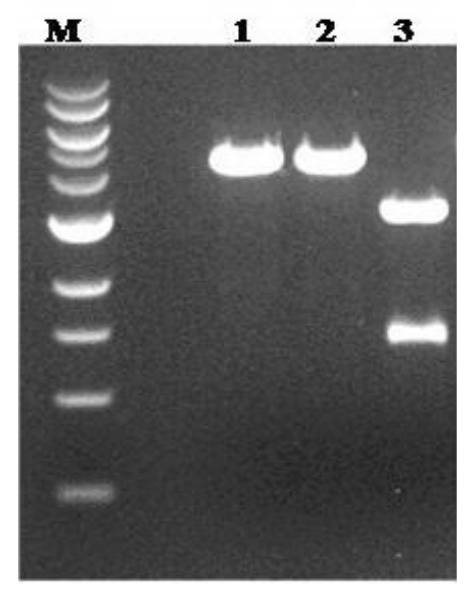

Figure 5. Restriction enzyme digestion of recombinant pGEM-T Easy plasmids with the cassava auxin repressedlike protein gene (ARP) promoter inserts. $\mathrm{M}=1-\mathrm{kb}$ ladder (sizes as shown in Figure 1); lane $1=\mathrm{ARP} /$ Bam HI; lane $2=\mathrm{ARP} /$ NcoI; lane $3=\mathrm{ARP} /$ BamHI and NcoI.

\section{Analysis of gene promoter sequences employing the PLACE database identified important promoter motifs}

Using the PLACE program (Higo et al., 1999), many regulatory elements known in other plants were identified in the ARP promoter sequence, such as light-responsive elements, ABA-responsive element, stress-responsive element, jasmonate- and elicitor-responsive elements, ACGT motif related to root expression and root-specific elements, among others (the motifs identified in the ARP promoter are listed in Table 1). Some of these regulatory elements have also been found in the cassava Mec1 promoter (de Souza et al., 2009) and in the C54 promoter sequence (Zhang et al., 2003). 
Isolation and partial characterization of cassava promoters

1039

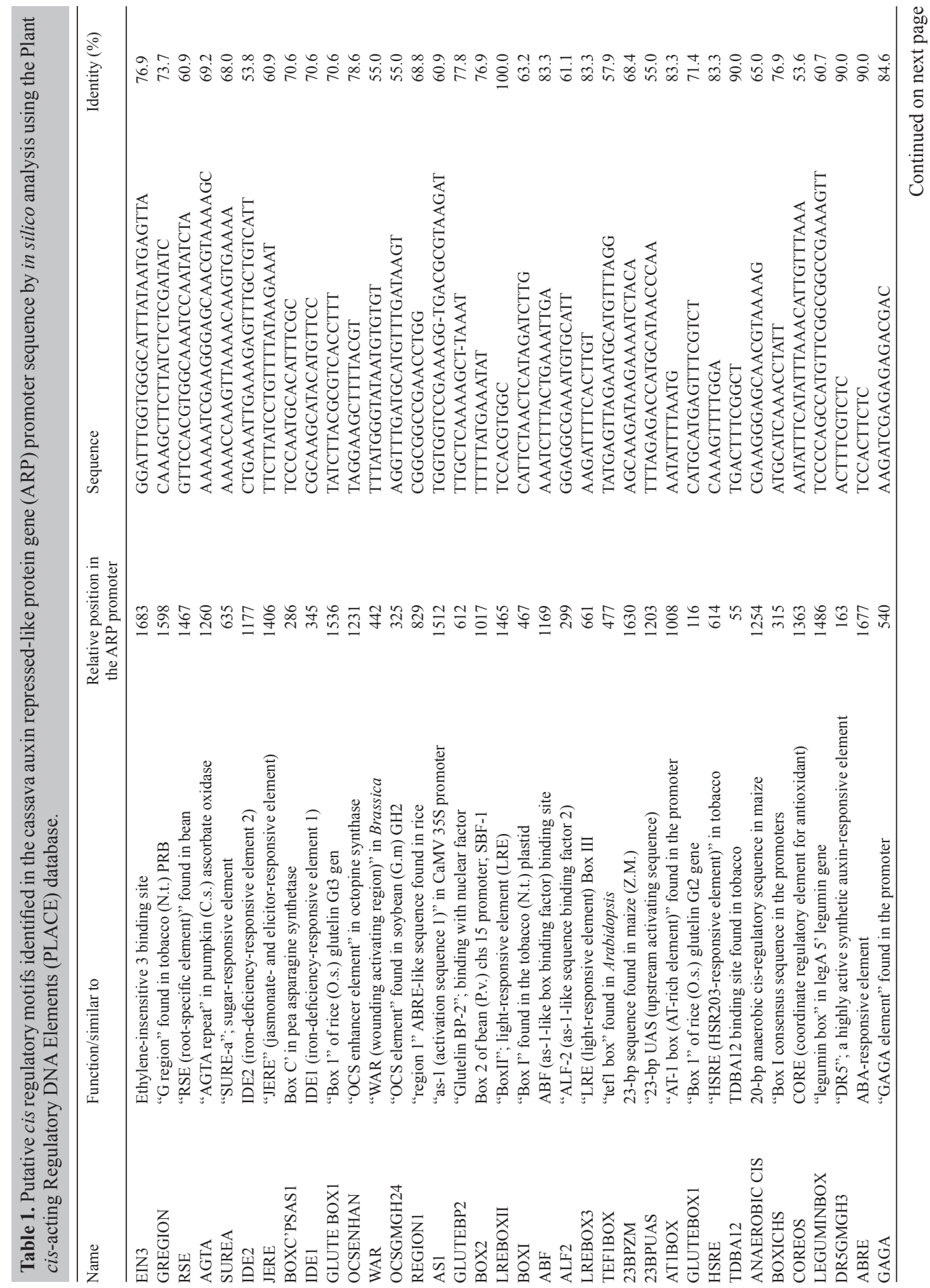

Genetics and Molecular Research 10 (2): 1032-1041 (2011)

CFUNPEC-RP www.funpecrp.com.br 


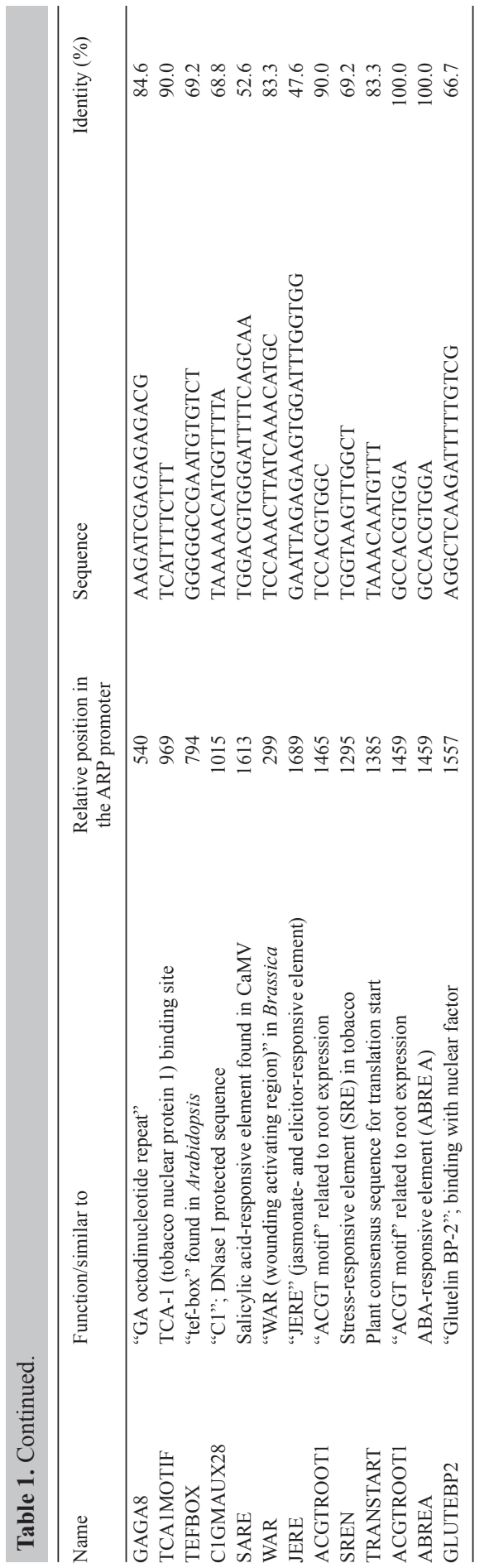




\section{ACKNOWLEDGMENTS}

Research supported by Rothamsted International, Harpenden, UK, under the African Fellowship Programme scheme.

\section{REFERENCES}

Altschul SF, Madden TL, Schaffer AA, Zhang J, et al. (1997). Gapped BLAST and PSI-BLAST: a new generation of protein database search programs. Nucleic Acids Res. 25: 3389-3402.

Beeching JR, Han Y, Gómez-Vásquez R, Day RC, et al. (1998). Wound and defense responses in cassava as related to post-harvest physiological deterioration. Recent Adv. Phytochem. 32: 231-248.

Cock JH (1985). Cassava: New Potential for a Neglected Crop. Westfield Press, Boulder.

de Souza CR, Aragao FJ, Moreira EC, Costa CN, et al. (2009). Isolation and characterization of the promoter sequence of a cassava gene coding for Pt2L4, a glutamic acid-rich protein differentially expressed in storage roots. Genet. Mol. Res. 8: 334-344.

Dellaporta SL, Wood J and Hicks JB (1983). A plant DNA minipreparation: version II. Plant Mol. Biol. Rep. 1: 19-21.

El-Sharkawy MA (2004). Cassava biology and physiology. Plant Mol. Biol. 56: 481-501.

Higo K, Ugawa Y, Iwamoto M and Korenaga T (1999). Plant cis-acting regulatory DNA elements (PLACE) database: 1999. Nucleic Acids Res. 27: 297-300.

Lu G and Moriyama EN (2004). Vector NTI, a balanced all-in-one sequence analysis suite. Brief. Bioinform. 5: 378-388.

Montagnac JA, Davis CR and Tanumihardjo SA (2009). Nutritional value of cassava for use as a staple food and recent advances for improvement. Compr. Rev. Food Sci. Food Safety 8: 181-194.

Puonti-Kaerlas J (1998). Cassava biotechnology. Biotechnol. Genet. Eng. Rev. 15: 329-364.

Restrepo S, Velez CM, Duque MC and Verdier V (2004). Genetic structure and population dynamics of Xanthomonas axonopodis pv. manihotis in Colombia from 1995 to 1999. Appl. Environ. Microbiol. 70: 255-261.

Sambrook J, Fritsch E and Maniatis T (1989). Molecular Cloning: A Laboratory Manual. Cold Spring Harbor Laboratory Press, Cold Spring Harbor.

Thomson JA (2008). The role of biotechnology for agricultural sustainability in Africa. Philos. Trans. R. C. Lond. B. Biol. Sci. 363: 905-913.

Verdier V, Restrepo S, Mosquera G, Jorge V, et al. (2004). Recent progress in the characterization of molecular determinants in the Xanthomonas axonopodis pv. manihotis-cassava interaction. Plant Mol. Biol. 56: 573-584.

Zhang P, Bohl-Zenger S, Puonti-Kaerlas J, Potrykus I, et al. (2003). Two cassava promoters related to vascular expression and storage root formation. Planta 218: 192-203. 